\title{
machinesMemory: Malleability of Al technique, the data generated by machine learning algorithms
}

\author{
Dongyuan Liu \\ Goldsmiths, University of London \\ 8 Lewisham Way, New Cross, London \\ SE14 6NW, UK \\ d.liu001@outlook.com
}

\begin{abstract}
machinesMemory explores the aesthetics of Al technology and its medium malleability. By the way of artistic practice, the author would like to focus on what Al technique is, rather than what it can do. This paper reviews the definition 'intelligent agents' to outline the basis of how machine learning algorithms will be used in this paper. This paper then introduces the making progress of machinesMemory and presents individual evaluations.
\end{abstract}

Machine learning. Intelligent agent. Machine generated data. Machine learning algorithm. Data visualisation.

\section{INTRODUCTION}

This paper presents practice-based research, which explores the malleability of artificial intelligence. The final output of this research is a multi-screen installation named machinesMemory in which the author uses an abstract way to express the idea of a machine's memory, and to apply its narrative in a structured installation.

In recent years, machine learning is a popular key phrase in artistic practices. Artists who are active in the field of Al-generated art, like Mario Klingemann, Gene Kogan and Sougwen Chung, created some inspiring artworks. They address the problems about what machine learning can do. The author, however, asks the question about what the machines create, what the machine-generated data are. Rather than considering the mathematic problem in a computer science field, the author, instead, would like to focus on the impact of $\mathrm{Al}$ technique on human society or machine society.

In this paper, the definitions and usages of machine learning in artistic projects will be reviewed to give readers a basic sense of the connection between human society and machine society. Then, the author considers data visualisation and image processes as methods to understand and express the characteristics of machine-generated data.

\section{BACKGROUND RESEARCH}

\subsection{Artistic perspective of machine learning}

\subsubsection{The definition of intelligent agents}

Both the terms 'autonomous agent' (Davidsson 1992) and 'intelligent agent' (Klicek 2010) indicate that $\mathrm{Al}$ agents should possess the ability to sense the environment via external sensors, to make decisions based on internal mechanisms, and to respond to the environment around them. Additionally, Blackwell presents four attributes of an entity's autonomy, whether it be human or machine. He especially focuses on the property of autonomy, which suggests that autonomous agents should be uncontrollable, unpredictable, and nonpredetermined (Blackwell 2012). Although machines do not, unlike humans, possess abilities granted by complex perceptive, sensitive, and cognitive neurons, machine learning enables them to act and react like humans. Boden frames her justification of the creative abilities of computer programs by analogy. The 'Lovelace questions' point out essential explorations of our understanding of how we recognise ourselves as humans in relation to machine beings (Boden 2004). Although she established a degree of similarity between humans and machines, the human qualities were ignored, as evidenced by the value of the artefacts presented. What is interesting is that we always explore how to make machines more human-like, but, in Boden's description, we 
can also understand ourselves by observing Al. We find that machine learning-related research studies have been premised upon comparing machines with humans. Machine learning, as a technique, gives machines the ability to perform like humans. When we criticise their creative abilities, we can do so using the language of the descriptions of human experience, such as how we paint, how we feel, and how we react. After all, the premise of the existence of artificial intelligence is to compare it with human capabilities.

\subsubsection{Machine learning in artistic practices}

In Drawing Operations Unit: Generation 2: Memory, by Sougwen Chung, and Neural Glitch, by Klingemann, the artists use $\mathrm{Al}$ algorithms to explore the aesthetic values of machine learning and determine whether computers can create works of art at the same level as humans.

Based upon the research mentioned above, the aesthetic value of machine learning can be roughly summarised as 'an unpredictable output of humanrelated resources', which is visually satisfying, a sense of order (AI and Neurography 2018). Similar to the question proposed by Klingemann, 'what a neural network has learned' (Al and Neurography 2018), Manovich (2018) describes machinegenerated data as a 'black box'. People do not know what the meanings of the numbers behind the images are, which can be easily transformed to images, sentences, or music. Getting access to the 'black box' may be an interesting topic in artistic practices.

The technique of deep learning is a sub-concept of machine learning and has emerged in a great deal of artistic projects. Recently developed processes of computational art use a combination of art and technology, and artists have been concentrating on what cutting-edge techniques, such as machine learning and big data, can offer their daily creative practices. When a state-of-the-art technique is developed, we often label it and explore the related characteristics within that label. The camera, for example, was labelled with 'reality', the Internet was labelled with 'connection', and machine learning was labelled with 'humanity'. Therefore, we can treat intelligent agents as we would a human when analysing them in the art world. Our discussions on the relationships between humans and machines, which are embedded into machine learning, are, in a way, an exploration of the relationships between humans.

\subsection{Data as material}

In The Language of New Media, Manovich (2002) coined the term 'info-aesthetic' as a cultural form, indicating that there are two such forms - database and 3D space - that can be found in new areas of media studies. The form of database, he explains as 'constructing the right interface to a multi-media database', can be easily found in most computational art works, whether based on a big database or a small database. In some data visualisation projects and some new media performances, artists collected data about, for example, body movements, weather information, and biological information to create visual patterns or sonic effects. In such cases, the artists know which number represents the left hand, and which number the right, and they use these data to present the beauty of those data.

Also, in the paper, 'How to compare one million images', the authors consider the comic database as a material to build a pattern which can be used to reflect some information and declare how the visual pattern connects to human culture (Manovich 2012). Data visualisation can reflect the information contained in a massive dataset by generating a graphic. However, from the viewpoint of artistic practice, it should not be used as it would for a business report. For example, a business report can reflect how many products were sold over the past several months and whether sales are up or down. Artists, however, would prefer to use meaningful data to produce a beautiful pattern or image that has a sense of order. For them, it is about how they understand the data (Evers et al. 2016).

The term 'material' is such a popular key word in artistic practices. Artists would like to find characteristics of materials, and then put them in an appropriate environment to present their narratives as languages. However, in the digital word, to present the narrative, we still need help with algorithms, or information processes that show the 'effect' in cause-and-effect (Manovich 2002).

\section{MAKING PROGRESS}

\subsection{Obtain the data}

To emphasise the independence of machines, I collected 1,000 photos from the photo albums of my friends' mobile phones that represent the idea of memory in an abstract way. I used this dataset to train the styleGAN deep learning model. After nine hours and the 1,000-step training, an abstract consequence resulted (Figure 1). Features from the original training set can be found in some machinegenerated images, but other parts of these generated images are rather abstract. Most of the images in the training set were landscape photos, hence some are recognisable (e.g. Italian landscapes). However, there are still some pictures 


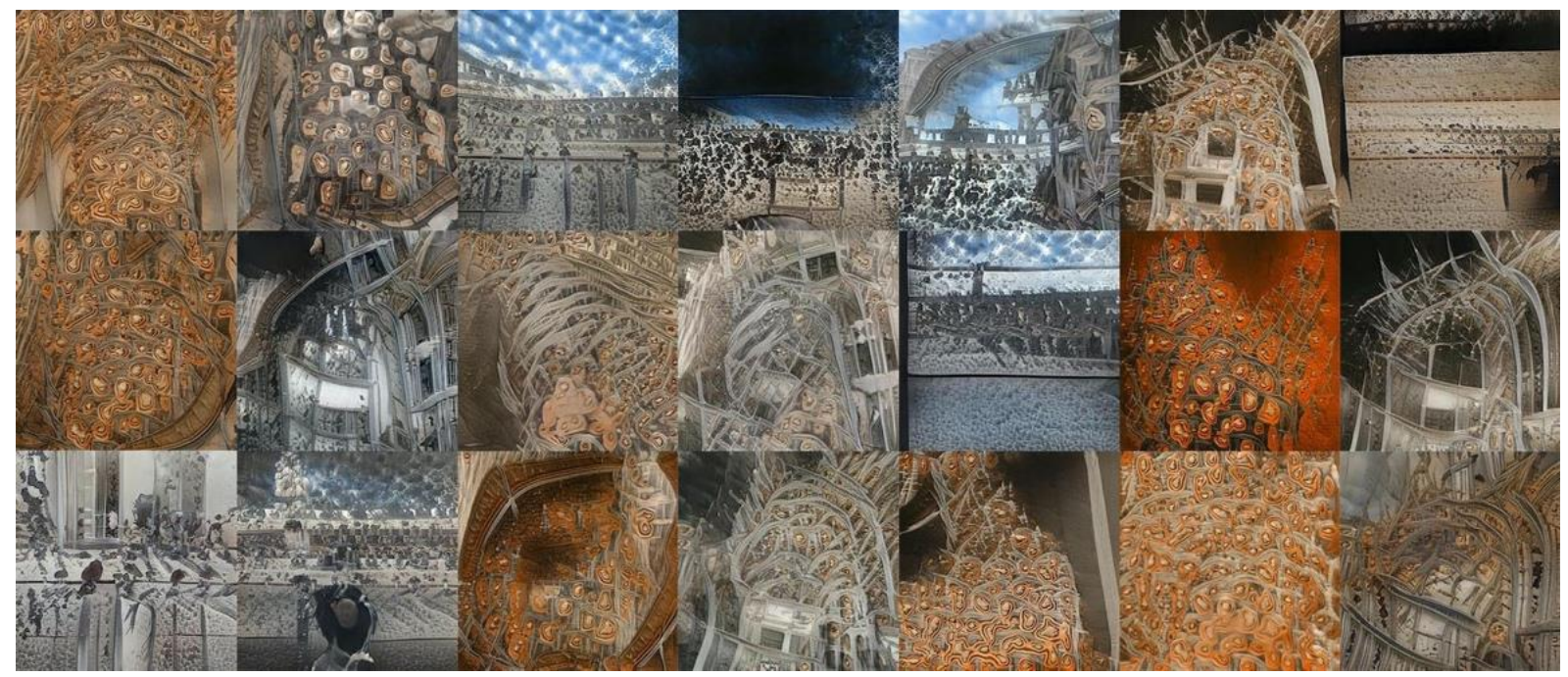

Figure 1: Dongyuan Liu, machinesMemory, multi-screens installation, 2020. Machine learning generated images (c) Dongyuan Liu).

that are unrecognisable but contain a sense of order. Machines certainly cannot possess such memories at the conscious level. However, from the perspective of ontology, we can imagine that these 'memories' do exist in some way.

The reason why I chose those photos from personal albums as training data is because I want to emphasise the diversity or complexity of the data in the human world, rather than the specific training set in a computer science field. For instance, a vehicle training set would like to be used to train a neural network that can generate vehicle-like images. In this research, we do not talk about what machine learning can do. Hence, it does not matter if the machine can learn those photos accurately or not.

\subsection{Information process}

Figure 2 shows the results of the feature-tracking image-processing algorithm. The red dots were extracted by an algorithm in OpenCV called goodFeatureToTrack. It seems like this yielded more meaningful data. The featured points can be thought of as analogous to feature information in human memories. For example, people tend to capture feature data when they remember a photo instead of remembering every pixel. As I mentioned in section 2.2, the OpenCV algorithm named goodFeatureToTrack is the tool, which can be used to show the 'effect' caused by the invisible data, which I also called 'a machine's way'.

After obtaining these feature points, I arranged them based on their brightness and hue values. As the brightness value becomes larger, the position of the point is more outward. As the hue value becomes larger, the rotation angle of the point becomes larger (Figure 3). Then, I spilt the feature points extracted from machine-generated images into different groups and assigned them an index under every sample. Every sample shows the pixel information from the corresponding picture. I refer to this as 'a machine's way' because people are still unable to extract these feature points actively; they were extracted by machines themselves, but we know what those data represented.

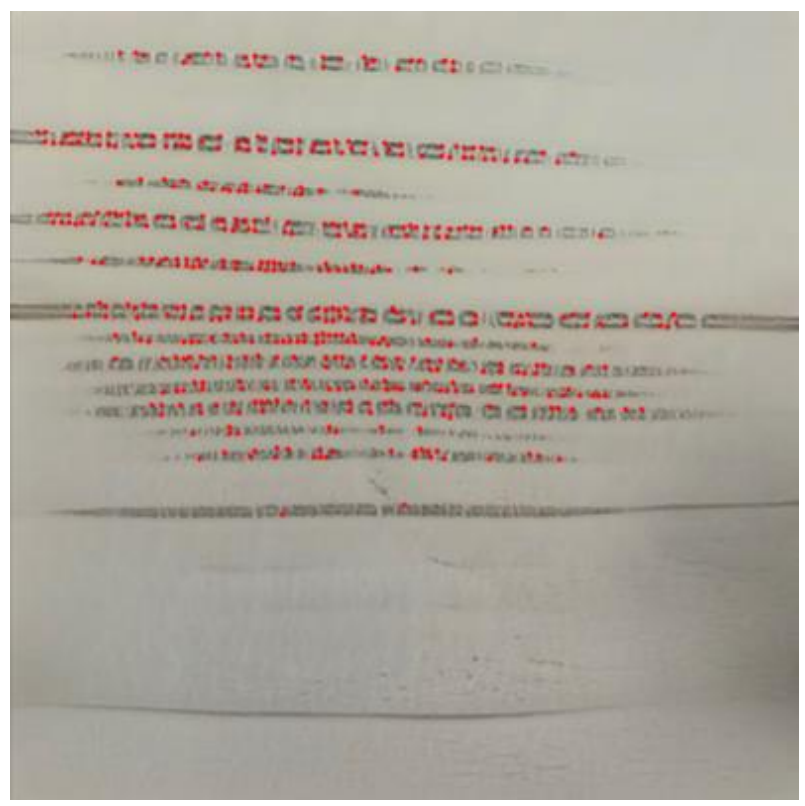

Figure 2: Dongyuan Liu, machinesMemory, multi-screens installation, 2020. Feature points (C) Dongyuan Liu). 


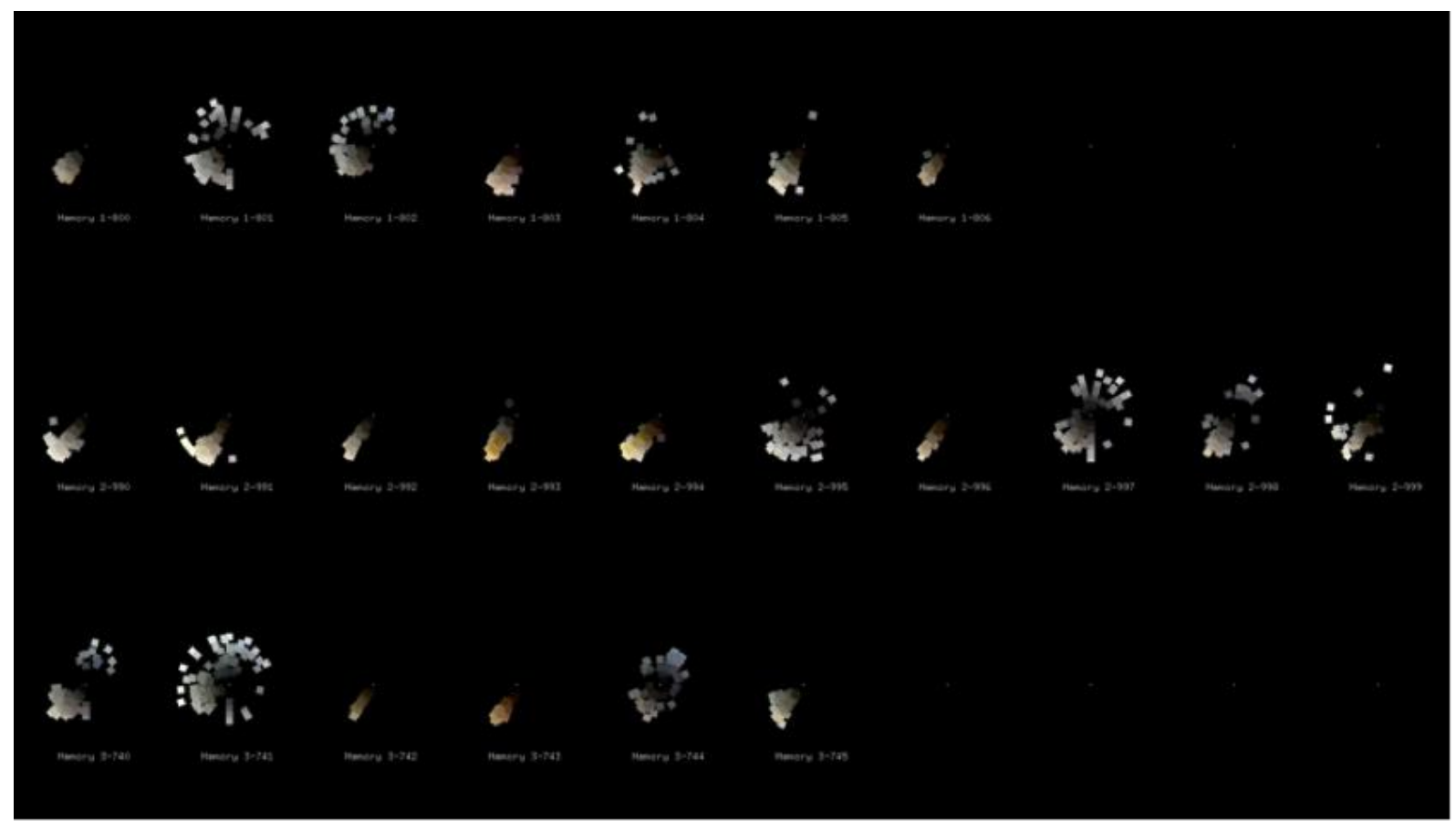

Figure 3: Dongyuan Liu, machinesMemory, multi-screens installation, 2020. Data visualisation (C Dongyuan Liu).

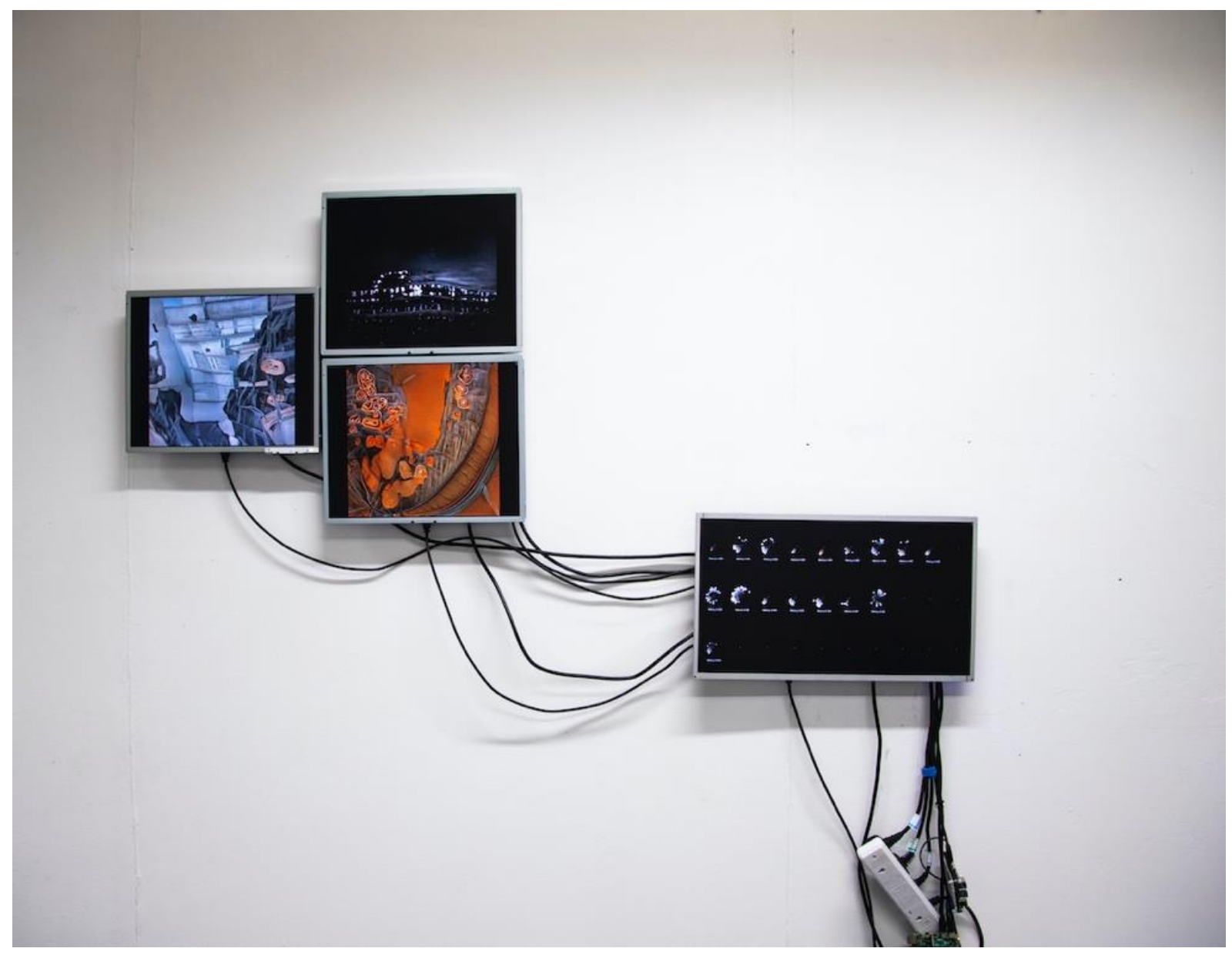

Figure 4: Dongyuan Liu, machinesMemory, multi-screens installation, 2020. Machine learning generated images (c) Dongyuan Liu). 


\subsection{Installation}

After obtaining a satisfactory result, I contemplated how to connect the various parts to present the idea of 'memory', which I intended to be nonlinear and complex. For narrative purposes, I used three screens, which were driven by three raspberry pi respectively, to function as the different areas representing nonlinear memory. Every machine contained thousands of different generated images that resulted from training based on different values for the parameters of the machine learning model. Then, I put them in openframework to calculate the 'goodFeature' points. The bottom one receives the extracted colour information by open sound control, and I arranged these data according to the aforementioned rules. Furthermore, the cables were used to connect the first, described in section 3.1, and second part, described in section 3.2.

\section{CONCLUSION}

The final output of this project is an abstract expression about Al technology. It was built based upon an emotional interpretation to emphasise the potential of its narrative in structured installation. The audiences were trying to make a connection between the generated images and the part of the data visualisation.

The topics related to machine learning on the conscious level still seem like unsolvable issues. However, from a narrative perspective, we may consider it as a way to express the cognition of machines, such as what the 'black box' contains. The making process of this practice is a preliminary exploration about how to understand the output of machine learning algorithms. machinesMemory proposed the topic of malleability of machine learning techniques in artistic practices. To be specific, it considers the method of data visualisation as a language to express the massive data generated by intelligent agents. In this practice, the algorithms used for information processes, the so called 'machine's way' are vital for the expression. The author wants to emphasise machine learning as a medium rather than the use of technology in artistic creation. This research would like to bring more possibilities for the interpretation of machine learning algorithms to understand the role of artificial intelligence in human social culture or machine social culture. For future work with the development of information process algorithms, artists would like to narratively explain the products of artificial intelligence more flexibly, and link them to the human social cultures.

\section{REFERENCES}

Blackwell, T. et al. (2012) 'Live Algorithms: Towards Autonomous Computer Improvisers'. Computers and Creativity, edited by Jon McCormack and Mark d'Inverno, Springer Berlin Heidelberg.

Boden (2004) Margaret A. The Creative Mind: Myths and Mechanisms. Routledge.

Chung, Sougwen. Sougwen Chung (愫君) - Works by Sougwen. https://sougwen.com/ (retrieved 6 June 2020).

Davidsson, P. (1992) Concept Acquisition by Autonomous Agents: Cognitive Modeling versus the Engineering Approach. Lund University Cognitive Studies 12, Lund University, Sweden. (PDF) Concepts and Autonomous Agents. Available from:

https://www.researchgate.net/publication/2910327 Concepts and Autonomous Agents

Evers, L., and Nack, F. (2016) 'Data Aesthetics: The Ethics and Aesthetics of Big Data Gathering Seen from the Artists Eye'. Proceedings of the 2016 ACM on Multimedia Conference - MM '16, ACM Press, 2016, pp.779-80.

Getting your Raspberry $\mathrm{Pi}$ ready for openFrameworks.

https://openframeworks.cc/setup/raspberrypi/raspb erry-pi-getting-started/ (retrieved 15 August 2020).

Klicek, B. (2010) 'Intelligent agents. International Encyclopedia of Hospitality Management, edited by Abraham Pizam, Routledge, 2nd edition.

Klingemann, M. (2018) 'Al and Neurography', Interalia Magazine.

https://www.interaliamag.org/interviews/marioklingemann/ (retrieved 14 July 2020).

Klingemann, M. (2020) 'Mario Klingemann, Artist Working with Code, Al and Data'. Quasimondo, http://quasimondo.com/ (retrieved 6 June 2020).

Kogan, G. (2020) genekogan.com, https://genekogan.com/ (retrieved 6 June 2020).

Manovich, L. (2002) The Language of New Media. 1st MIT Press Paperback ed., MIT Press.

Manovich, L. (2012) 'How to Compare One Million Images?' In David Berry, ed., Understanding Digital Humanities.

Manovich, L. (2018) Al Aesthetics, 2018. http://www.manovich.net.

PyData (2020) 'Keynote: The Neural Aesthetic Gene Kogan'. PyData, YouTube, https://www.youtube.com/watch?v=XPqCITbl3RE. (retrieved 15 March 2020).

StyleGan (2020) StyleGan Github. https://github.com/NVlabs/stylegan (retrieved 12 August 2020). 